\title{
CONVERGENCE OF SEQUENCES OF SEMIGROUPS OF NONLINEAR OPERATORS WITH AN APPLICATION TO GAS KINETICS $\left({ }^{1}\right)$
}

\author{
BY
}

THOMAS G. KURTZ

ABSTRACT. Let $A_{1}, A_{2}, \cdots$ be dissipative sets that generate semigroups of nonlinear contractions $T_{1}(t), T_{2}(t) \ldots$. Conditions are given on $\left\{A_{n}\right\}$ which imply the existence of a limiting semigroup $T(t)$. The results include types of convergence besides strong convergence.

As an application, it is shown that solutions of the pair of equations

$$
u_{t}=-a_{u_{x}}+a^{2}\left(v^{2}-u^{2}\right)
$$

and

$$
v_{t}=a v_{x}+a^{2}\left(u^{2}-v^{2}\right)
$$

a a constant, approximate the solutions of

$$
u_{t}=1 / 4\left(d^{2} / d x^{2}\right) \log u
$$

as a goes to infinity.

1. Introduction. A general theorem concerning the convergence of sequences of semigroups of linear operators was given in [5]. The basis of the proof was the following corollary to the Hille-Yosida theorem.

Proposition (1.1). Let $T(t)$ be a strongly continuous semigroup of linear operators on a Banach space $L$ with infinitesimal operator $A$. Let $M$ be a closed subspace of $L$. If $(\lambda-A)^{-1}: M \rightarrow M$ for all $\lambda$ sufficiently large, then $T(t): M \rightarrow M$

Crandall and Liggett [3] have developed a theory for semigroups of nonlinear operators generated by accretive sets that implies essentially the same result. Consequently, many of the results in [5] can now be carried over to nonlinear semigroups of this type.

Received by the editors October 5, 1972 and, in revised form, April 10, 1973. AMS (MOS) subject classifications (1970). Primary 47H15; Secondary 34G05, 82 A40.

Key words and phrases. Nonlinear semigroups, approximation, gas kinetics.

(1) Sponsored by the United States Army under Contract No. DA-31-124-AROA-462. 
In $\$ 2$, we state the relevant results of Crandall and Liggett and develop the necessary background for the convergence theorem in $\$ 3$. In $\$ 4$ we consider the corresponding discrete parameter convergence theorem. In $\$ 5$, we consider an example arising in gas kinetics and obtain a limit theorem analogous to a result of Pinsky [11] for a related linear problem.

2. Background. We state the results of Crandall and Liggett [3] in terms of dissipative rather than accretive sets so they will look more like the corresponding results in the linear case.

Let $L$ be a Banach space. If $A \subset L \times L$ define the domain of $A$ by

$$
\mathcal{D}(A)=\{x:(x, y) \in A \text { for some } y\}
$$

the range of $A$ by

$$
R(A)=\{y:(x, y) \in A \text { for some } x\}
$$

the inverse of $A$ by

$$
A^{-1}=\{(y, x):(x, y) \in A\}
$$

and scalar multiplication by

$$
a A=\{(x, a y):(x, y) \in A\} .
$$

If $A \subset L \times L$ and $B C L \times L$ then define

$$
A+B=\left\{\left(x, y_{1}+y_{2}\right):\left(x, y_{1}\right) \in A\left(x, y_{2}\right) \in B\right\} \text {. }
$$

Note that $\mathscr{D}(A+B)=\mathscr{D}(A) \cap \mathcal{D}(B)$. For fixed $x, A x$ denotes $\{y:(x, y) \in A\}$.

If $A_{1}, A_{2} \cdots$ is a sequence of subsets of $L \times L$ then

$$
\lim _{n \rightarrow \infty} A_{n} \equiv \bigcap_{\epsilon} \bigcup_{m} \bigcap_{n>m} A_{n, \epsilon}
$$

where

$$
A_{n, \epsilon} \equiv\left\{(\hat{x}, \hat{y}): \exists(x, y) \in A_{n}\|x-\hat{x}\|,\|y-\hat{y}\| \leq \epsilon\right\} .
$$

This definition of the limit of a sequence of sets is equivalent to saying $(x, y) \epsilon$ $\lim _{n \rightarrow \infty} A_{n}$ if and only if there are $\left(x_{n}, y_{n}\right) \in A_{n}$ such that $\lim _{n \rightarrow \infty}\left(\left\|x-x_{n}\right\|+\left\|y-y_{n}\right\|\right)$ $=0$. A set $A \subset L \times L$ is called dissipative if $\left(x_{1}, y_{1}\right),\left(x_{2}, y_{2}\right) \in A$ implies

$$
\left\|\left(x_{1}-\alpha y_{1}\right)-\left(x_{2}-\alpha y_{2}\right)\right\| \geq\left\|x_{1}-x_{2}\right\|
$$

for all $\alpha>0$.

It follows from (2.1) that $I-a A$ has a single valued inverse satisfying

$$
\left\|(I-\alpha A)^{-1} x-(I-\alpha A)^{-1} y\right\| \leq\|x-y\|
$$

for $x, y \in R(I-\alpha A), a>0$. 
Theorem (2.3) (Crandall and Liggett). Let $A \subset L \times L$ and $w$ be a real number such that $A-w I$ is dissipative. If $R(I-\alpha A) \supset \overline{D(A)}$ for all sufficiently small positive $a$, then for all $x \in \overline{\mathfrak{D}(A)}$

$$
S(t) x \equiv \lim _{m \rightarrow \infty}\left(1-\frac{t}{m} A\right)^{-m} x
$$

exists; $S(t) x$ is a strongly. continuous function of $t ; S(t+s) x=S(t) S(s) x$; and

$$
\|S(t) x-S(t) y\| \leq e^{w t}\|x-y\|
$$

all $x, y \in \overline{\mathscr{D}(A)}$.

Corollary (2.5). If $C$ is a closed subset of $\overline{\mathscr{D}(A)}$ and $(I-a A)^{-1}: C \rightarrow C$ for all sufficiently small positive $\alpha$, then

$$
s(t): C \rightarrow C, \text { all } t>0 .
$$

A corollary to the main limit theorem (Theorem (3.2)) we will prove is the following:

Theorem (2.6). Let $\left\{A_{n}\right\}$ be a sequence of subsets of $L \times L$ satisfying the conditions of Theorem (2.3) with a common $w$, and let $\left\{T_{n}(t)\right\}$ be the correspond. ing sequence of semigroups. Define $A=\lim _{n \rightarrow \infty} A_{n^{\circ}}$ Then $A-w I$ is dissipative and if $\overline{R(I-\alpha A)} \supset \overline{\mathscr{D}(A)}$ for all sufficiently small positive $a$, then (by Theorem (2.3)). A generates a semigroup $T(t)$ and

$$
T(t) x=\lim _{n \rightarrow \infty} T_{n}(t) x_{n}
$$

for all $x \in \overline{\mathscr{D}(A)}$ and all sequences $x_{n} \in \overline{\mathfrak{D}\left(A_{n}\right)}$ with $\lim _{n \rightarrow \infty} x_{n}=x$.

We actually will prove a more general abstract theorem which will apply to notions of convergence other than strong convergence, as well as to "convergence" of semigroups defined on different Banach spaces in the manner introduced by Trotter [12]. To motivate the abstract formulation consider the following:

Let $\mathcal{L}$ be the Banach space of bounded sequences $\left\{x_{n}\right\} \subset L$ with $\left\|\left\{x_{n}\right\}\right\| \equiv$ $\sup _{n}\left\|x_{n}\right\|$. For $A_{1}, A_{2} \ldots$ satisfying the conditions of Theorem (2.3) with common $w$ let

$$
\mathfrak{Q}=\left\{\left(\left\{x_{n}\right\},\left\{y_{n}\right\}\right):\left(x_{n}, y_{n}\right) \in A_{n} ;\left\{x_{n}\right\},\left\{y_{n}\right\} \in £\right\} .
$$

Then $\mathfrak{C} \subset \mathfrak{L} \times \mathfrak{L}$ satisfies the conditions of Theorem (2.3). Consequently,

$$
\mathcal{T}(t)\left\{x_{n}\right\} \equiv \lim _{m \rightarrow \infty}\left(I-\frac{t}{m} \mathfrak{Q}\right)^{-m}\left\{x_{n}\right\}
$$

exists for all $\left\{x_{n}\right\} \in \overline{\mathscr{T}(\mathscr{Q})}$ and $\mathscr{T}(t)\left\{x_{n}\right\}=\left\{T_{n}(t) x_{n}\right\}$, where $T_{n}(t)$ is the semigroup corresponding to $A_{n}$. Let $\pi \subset \mathcal{L}$ be the subspace of strongly convergent sequences. If 


$$
\mathscr{T}(t): \mathbb{M} \cap \overline{\mathscr{D}(\mathscr{Q})} \rightarrow \pi \cap \overline{\mathscr{D}(\mathscr{Q})}
$$

then defining

$$
T(t) x \equiv \lim _{n \rightarrow \infty} T_{n}(t) x_{n},
$$

whenever $\left\{x_{n}\right\} \in \mathbb{M} \cap \overline{\mathfrak{D}(\mathfrak{Q})}$ and $x=\lim _{n \rightarrow \infty} x_{n}$, we obtain a strongly continuous semigroup on $\left\{x: x=\lim _{n \rightarrow \infty} x_{n},\left\{x_{n}\right\} \in M \cap \cap \bar{D}(\mathfrak{Q})\right\}$ satisfying $\|T(t) x-T(t) y\| \leq$ $e^{w t}\|x-y\|$. This expains our interest in Corollary (2.5) and motivates the following abstract formulation of the problem which will be considered in $\$ 3$.

Let $\mathcal{L}$ and $L$ be Banach spaces, and let $P$ be a bounded linear mapping defined on a closed subspace $\mathcal{D}(P)$ of $\mathscr{L}$,

$$
P: \mathscr{D}(P) \rightarrow L .
$$

Let $\mathfrak{Q} \subset \mathfrak{L} \times \mathfrak{L}$ satisfy the conditions of Theorem (2.3) and let $\mathscr{T}(t)$ be the corresponding semigroup. Under what conditions does

$$
T(t) P x \equiv P \mathcal{T}(t)_{x}, \quad x \in \overline{\mathscr{D}(\mathscr{Q})} \cap \mathcal{D}(P),
$$

determine a semigroup on a subset of $L$ ? In the case of primary interest $\mathscr{L}$ is the sequence space and $P\left\{x_{n}\right\}=\lim _{n \rightarrow \infty} x_{n}$, the limit being the strong limit. However, note that other notions of convergence (e.g. weak convergence) determine bounded linear operators on subspaces of the sequence space in exactly the same way.

Similarly in Trotter's setting we have a Banach space $L$, a sequence of Banach spaces $L_{n}$, and linear maps $P_{n}: L \rightarrow L_{n}$ satisfying $\lim _{n \rightarrow \infty}\left\|P_{n} x\right\|=\|x\|$. Let $\mathcal{L}=\left\{\left\{x_{n}\right\}: x_{n} \in L_{n}\left\|\left\{x_{n}\right\}\right\| \equiv \sup _{n}\left\|x_{n}\right\|<\infty\right\}$. Define $P\left\{x_{n}\right\}=x$ if $\lim _{n \rightarrow \infty}\left\|P_{n} x-x_{n}\right\|=0$. This defines a bounded linear operator on a subspace of $\mathscr{L}$.

When this paper was in its final draft, the author learned that Professor Jerome A. Goldstein [14] had proved a theorem virtually identical to Theorem (2.6) using a somewhat different sequence space approach. For most purposes Theorem (2.6) is also equivalent to the results of Brezis and Pazy [1] which in turn generalize results in [7], [8], [9], [10]. Most of this work assumes convergence of $\left(I-\alpha A_{n}\right)^{-1}$ in some sense rather than convergence of $A_{n}$. Conditions of one type can of course be translated into conditions of the other type.

\section{Transformation of semigroups.}

Lemma (3.1). Let $\mathcal{Q}$ and $L$ be Banach spaces. Let $P$ be a continuous linear mapping of a closed subspace $\mathscr{D}(P) \subset \mathfrak{Q}$ into $L$ and let $\mathfrak{B}$ be a Lipschitz continuous (but not necessarily linear) mapping of a closed subset $\mathscr{D}(\mathfrak{B}) \subset \mathfrak{L}$ into $\mathfrak{\&}$.

Let $D=\mathfrak{D}(\mathfrak{B}) \cap \mathscr{D}(P)$.

For $y_{1}, y_{2} \in P(D)$ define $\rho\left(y_{1}, y_{2}\right)=\inf \left\{\left\|x_{1}-x_{2}\right\|: x_{1}, x_{2} \in D, y_{1}=P x_{1}, y_{2}=\right.$ $\left.P x_{2}\right\}$ and suppose there is a constant $c$ such that $\rho\left(y_{1}, y_{2}\right) \leq c\left\|y_{1}-y_{2}\right\|$ all $y_{1}, y_{2} \epsilon$ $P(D)$. 
Suppose $x, y \in D$, and $P x=P y$ implies $B_{x}-B_{y} \in \mathscr{D}(P)$ and $P\left(\mathfrak{B} x-B_{y}\right)=0$. Let $\mathcal{C} \equiv\left\{x \in D: B_{x} \in \mathfrak{D}(P)\right\}$ and let $C$ be the closure of $P(\mathcal{C})$. Then $\mathcal{C}=$ $\{x \in D: P x \in C\}$, and $B P x \equiv P B x, x \in \mathcal{C}$ determines a Lipschitz continuous mapping $B$ of $C$ into $L$.

Proof. If $x \in D$ and $P x \in C$, then there is a sequence $\left\{z_{n}\right\} \subset \mathcal{C}$ with $\lim _{n \rightarrow \infty} P z_{n}=P x$. Using the assumptions on $\rho\left(y_{1}, y_{2}\right)$ we can find sequences $\left\{y_{n}\right\}$ and $\left\{x_{n}\right\}$ in $D$ such that $P y_{n}=P z_{n}, P x_{n}=P x$ and $\lim _{n \rightarrow \infty}\left\|y_{n}-x_{n}\right\|=0$. The fact that $B z_{n} \in \mathscr{D}(P)$ and the assumptions on $P$ and $\mathscr{B}$ imply $\mathfrak{B y}_{n}$ and $\mathfrak{B x}_{n}-$ $B_{x}$ are in $\mathscr{D}(P)$.

The Lipschitz continuity of $\mathfrak{B}$ implies $\lim _{n \rightarrow \infty} \mathfrak{B y}_{n}-\mathfrak{B x}_{n}=0$, and hence

$$
\lim _{n \rightarrow \infty}\left(\mathfrak{B y}_{n}-\mathfrak{B}_{n}+\mathfrak{B}_{x}\right)=\lim _{n \rightarrow \infty}\left(\mathfrak{B}_{n}-\left(\mathfrak{B}_{n}-\mathfrak{B}_{x}\right)\right)=\mathfrak{B}_{x}
$$

Since $B_{n}-\left(B_{n}-B_{x}\right) \in \mathscr{D}(P)$ we must have $B_{x} \in \mathscr{D}(P)$, and hence $x \in \mathcal{C}$. (Note we have not shown that $P(\mathcal{C})$ is closed.)

The Lipschitz continuity of $B$ follows from the Lipschitz continuity of $\mathfrak{B}$, the fact that $B$ is well defined, and the assumptions on $\rho\left(y_{1}, y_{2}\right)$.

Theorem (3.2). Let $\mathcal{L}$ and $L$ be Banach spaces, and let $P$ be a continuous linear mapping of a closed subspace $\mathscr{D}(P) \subset \mathcal{L}$ into $L$.

Let $\mathfrak{Q} \subset \mathfrak{Q} \times \mathfrak{Q}$ satisfy the conditions of Theorem (2.3) and denote the corresponding semigroup by $\mathcal{T}(t)$. Suppose the conditions of Lemma (3.1) are satis. fied taking $\mathfrak{B}=\mathfrak{T}(t)$ or $\mathfrak{B}=(I-a \mathfrak{P})^{-1}$. (This is always true under the conditions of Theorem (2.6).) Define

$$
A=\left\{\left(P_{x}, P_{y}\right):(x, y) \in \mathbb{Q} \cap \mathscr{D}(P) \times \mathscr{D}(P)\right\} .
$$

If $\overline{R(I-\alpha A)} \supset \overline{\mathscr{D}(A)}$ for all sufficiently small positive $a$, then $(I-\alpha A)^{-1}$ can be extended to a Lipscbitz continuous map $J(a)$ on $\overline{R(I-\alpha A)}$;

$$
(I-a A)^{-1} y=P(I-\alpha \mathfrak{C})^{-1} x
$$

if $y=P x \in \mathscr{R}(I-a A)$;

$$
T(t) y=P \mathcal{T}(t) x
$$

for $y=P_{x} \in \overline{\mathscr{D}(A)}$ determines a strongly continuous semigroup of Lipschitz continuous mappings on $\overline{\mathfrak{D}(A)}$; and

$$
T(t) x=\lim _{n \rightarrow \infty} J\left(\frac{t}{n}\right)^{n} x
$$

for $x \in \overline{\mathfrak{D}(A)}$. 
Proof. Let $D_{\alpha}=R(I-\alpha \mathfrak{Q}) \cap \mathscr{D}(P)$ and $D=\overline{\mathscr{D}(\mathfrak{Q})} \cap \mathscr{D}(P)$. By Lemma (3.1)

$$
\mathcal{C}_{\alpha}=\left\{x \in D_{\alpha}:(I-\alpha \mathfrak{Q})^{-1} x \in \mathcal{D}(P)\right\}=\left\{x \in D_{\alpha}: P x \in C_{\alpha}\right\},
$$

where $C_{\alpha}$ is the closure of $P\left(C_{\alpha}\right)$. Observe that

$$
c_{a} \supset \overline{\mathscr{R}(I-\alpha A)} \supset \overline{\mathscr{D}(A)}
$$

and

$$
(I-\alpha \mathfrak{Q})^{-1}: \mathcal{C}_{a} \rightarrow\left\{x \in D: P_{x} \in \overline{\mathscr{D}(\mathfrak{Q})}\right\} \equiv \mathcal{C} \subset \mathcal{C}_{a^{*}}
$$

It follows that

$$
(I-\alpha A)^{-1} P x=P(I-\alpha \mathscr{Q})^{-1} x
$$

for $x \in \mathcal{C}_{a}$ and $(I-a A)^{-1}$ can be extended to a Lipschitz continuous map on $\overline{R(I-\alpha A)}$. Since $(I-\alpha \mathscr{Q})^{-1}: \mathcal{C} \rightarrow \mathcal{C}$, Corollary (2.5) implies

$$
\mathcal{T}(t): \mathcal{C} \rightarrow \mathcal{C} .
$$

Again applying Lemma (3.1),

$$
\{x \in D: \mathscr{T}(t) x \in \mathcal{D}(P)\}=\left\{x \in D: P x \in C_{t}\right\}
$$

where $C_{t}$ is a closed subset of $L$. By (3.6) $C_{t} \supset \mathcal{D}(A)$ and (3.4) follows. The limit in (3.5) follows from (3.3) and the corresponding limit for $\mathcal{T}(t)$.

In the linear case the converse of Theorem (3.2) holds as a result of the fact that the converse of Corollary (2.5) holds. Unfortunately, due to the nonuniqueness of the dissipative set corresponding to a nonlinear semigroup (see [3]), counterexamples to the converse exist. On the positive side, the following can also be found in [3].

Proposition (3.7). Let $\mathfrak{Q}$ satisfy the conditions of Theorem (2.3), and $\overline{\mathscr{D}(\mathfrak{Q})}$ be convex. If

$$
\lim _{t \rightarrow 0_{+}}\left(I-\alpha\left(\frac{\mathcal{T}(t)-I}{t}\right)\right)^{-1} x=(I-\alpha \mathfrak{C})^{-1} x
$$

for all $x \in \overline{\mathfrak{D}(\mathfrak{Q})}$, then $\mathfrak{T}(t): \mathcal{C} \rightarrow \mathcal{C}, \mathcal{C}$ a closed convex subset of $\overline{\mathfrak{D}(\mathbb{Q})}$, implies $(I-a \mathfrak{Q})^{-1}: \mathcal{C} \rightarrow \mathcal{C}$.

We note that (3.8) is equivalent to

$$
\lim _{t \rightarrow 0+} \mathfrak{A}_{t}=\mathfrak{A} \cap \bigcup_{a}\left\{\left((I-a \mathfrak{Q})^{-1} x, x\right): x \in \overline{\mathscr{D}(\mathfrak{Q})}\right\}
$$

where 


$$
Q_{t}=\{(x,(\mathcal{T}(t)-I) x / t): x \in \overline{\mathscr{D}(\mathscr{C})}\},
$$

and the limit is defined in the same way as the limit of a sequence of sets was in $\$ 2$.

4. Discrete parameter limit theorem. In the linear case [4], [5], [12], discrete parameter limit theorems are obtained by comparing the sequence of discrete parameter semigroups $\left\{T_{n}(k)\right\}$ with a corresponding sequence of continuous parameter semigroups generated by

$$
A_{n}=\left\{\left(x,\left(T_{n}(1)-I\right) x / b_{n}\right): x \in \mathscr{D}\left(T_{n}(1)\right)\right\}
$$

where $\left\{b_{n}\right\}$ is an appropriately chosen sequence of positive numbers with $\lim _{n \rightarrow \infty} b_{n}=0$. The following, an immediate consequence of results due to Miyadera and Oharu [10], gives the nonlinear analogue.

Theorem (4.1). Let $\left\{T_{n}\right\}$ be a sequence of contractions with $R\left(T_{n}\right) \subset \mathcal{D}\left(T_{n}\right)$. Let $T_{n}(k)$ denote the $k$ th power of $T_{n}$ and define

$$
A_{n}=\left\{\left(x, b_{n}^{-1}\left(T_{n}-I\right) x\right): x \in \mathcal{D}\left(T_{n}\right)\right\},
$$

where $\lim _{n \rightarrow \infty} b_{n}=0$. Let $S_{n}(t)$ denote the contraction semigroup generated by $A_{n}$. If $x_{n} \in \mathscr{D}\left(T_{n}\right) \equiv \mathscr{D}\left(A_{n}\right)$ satisfies $\sup _{n}\left\|A_{n} x_{n}\right\|<\infty$, then

$$
\left\|S_{n}(t) x_{n}-T_{n}\left(\left[t / b_{n}\right]\right) x_{n}\right\| \leq b_{n}\left(b_{n}+\sqrt{t}\right)\left\|A_{n} x_{n}\right\|
$$

and bence

$$
\lim _{n \rightarrow \infty}\left\|S_{n}(t) x_{n}-T_{n}\left(\left[t / b_{n}\right]\right) x_{n}\right\|=0 .
$$

As a consequence of the above we state the following analogue of Theorem (2.G).

Theorem (4.2). Let $\left\{T_{n}\right\}$ and $\left\{A_{n}\right\}$ be as in Theorem (4.1) and define $A=$ $\lim _{n \rightarrow \infty} A_{n^{*}}$ Then $A$ is dissipative and if $\overline{R(I-\alpha A)} \supset \overline{\mathcal{D}(A)}$ for all sufficiently small positive $a$, then $A$ generates a semigroup $T(t)$ and

$$
T(t) x=\lim _{n \rightarrow \infty} T_{n}\left(\left[t / b_{n}\right]\right)_{n}
$$

for all $x \in \overline{\mathscr{D}(A)}$ and all sequences $x_{n} \in \mathscr{D}\left(T_{n}\right)$ with $\lim _{n \rightarrow \infty} x_{n}=x_{\text {. }}$

5. An example from gas kinetics. In [2] $\mathrm{H}$. Conner considers a number of discrete velocity models related to the Boltzmann equation. One of these, due to Carleman,

$$
u_{t}=-u_{x}+v^{2}-u^{2}, \quad v_{t}=v_{x}+u^{2}-v^{2}
$$

corresponds to a semigroup generated by a dissipative set. This can be considered as a model for a system with two types of particles, Type I particles move to the 
right on the line with speed one, Type II to the left with speed one. The local density of Type I particles is given by $u$ and that of Type II particles by $v$. When two particles interact one Type I particle is produced and one Type II particle. Consequently an interaction between a Type I particle and a Type II particle has no effect on the system.

The results of this paper allow us to give a rigorous derivation of the associated fluid equation. The fluid equation corresponds roughly to the behavior of the total density $\rho=u+v$ when the local velocity distribution is in equilibrium (in this case $u=v$ ). This can only be approximately true (unless $u \equiv v \equiv$ constant) but the approximation may be good if the local approach to velocity equilibrium is sufficiently rapid.

To clarify what we mean by this, define

$$
A(u, v)=\left(-u_{x}, v_{x}\right) \text { and } B(u, v)=\left(v^{2}-u^{2}, u^{2}-v^{2}\right) \text {. }
$$

Let $L=L_{1}(\mathbf{R}) \times L_{1}(\mathbf{R})$ with $\|(u, v)\|=\|u\|_{L_{1}}+\|v\|_{L_{1}}$, and $L^{+}=\{(u, v) \in L$ : $u \geq 0, v \geq 0\}$. Then $A$ and $B$ are defined and dissipative on a dense subset of $L^{+}$as is $A+a B$ for $a>0$, and the closure of $A+a B$ generates a semigroup $T_{\alpha}(t)$ in the sense of Theorem (2.3). (See Appendix.)

If $\alpha$ is large then the approach to equilibrium by the local velocity distribution is rapid. Consequently we are interested in the behavior of $T_{\alpha}(t)$ as a goes to infinity. We will actually consider $T_{\alpha}\left(\alpha_{t}\right)$ which is the semigroup corresponding to $\alpha A+\alpha^{2} B$.

Theorem (5.1). Let $T_{a}(t)$ be as defined above. Let $u \geq 0$ and $u \in L_{1}(\mathbf{R})$. Then

$$
\lim _{a \rightarrow \infty} T_{a}(a t)(u, u)=(T(t) u, T(t) u)
$$

where $T(t)$ is the semigroup on $\left\{u \in L_{1}(R): u \geq 0\right\}$ generated by the closure in $L_{1}(\mathbf{R})$ of

$$
C u=\frac{1}{4} \frac{d}{d x}\left(\frac{u_{x}}{u}\right)=\frac{1}{4} \frac{d^{2}}{d x^{2}} \log u,
$$

with $\mathscr{D}(C)=\left\{u \in L_{1}(\mathbf{R}): u>0, u\right.$ and $u_{x}$ absolutely continuous, $\left(d^{2} / d x^{2}\right) \log u \epsilon$ $L_{1}(\mathrm{R})$ and $\left.\lim _{|x| \rightarrow \infty} u_{x} / u=0\right\}$.

Proof. Let $\mathcal{L}$ be the space of mappings of $[0, \infty)$ into $L$ with norm

$$
\left\|\left\{\left(u_{a}, v_{a}\right)\right\}\right\|=\sup _{a}\left\|\left(u_{a}, v_{a}\right)\right\| \text {. }
$$

Define a bounded linear operator from a subspace of $\mathscr{Q}$ into $L_{1}(\mathbf{R})$ by

$$
P\left\{\left(u_{a}, v_{\alpha}\right)\right\}=u \text { if } \lim _{a \rightarrow \infty} u_{a}=u \text { and } \lim _{a \rightarrow \infty} v_{a}=u \text {. }
$$


To apply Theorem (3.2) we must find $\left\{\left(u_{\infty}, v_{a}\right)\right\} \in £$ such that

$$
P\left\{\left(u_{\alpha}, v_{\alpha}\right)\right\}=u \text { and } P\left\{\left(\alpha A+\alpha^{2} B\right)\left(u_{\alpha}, v_{\alpha}\right)\right\}=C u
$$

for sufficiently many $u$. Toward that end we prove the following lemma:

Lemma (5.3). Let $u$ be twice continuously differentiable, $\left(d^{2} / d x^{2}\right) \log u \epsilon$ $L_{1}(\mathbf{R}), u>0, c_{1} / x^{2} \leq u \leq c_{2} / x^{2}$ for some $c_{1}$ and $c_{2}$ and $x$ sufficiently large, and $\left|u_{x}\right| \leq\left|M / x^{3}\right|$ for some $M$.

Let $x_{a}=\inf \left\{|x|:\left|u_{x}(x)\right| \geq 4 a|u(x)|^{2}\right\}$ and define $g_{a}$ so that

$$
g_{a}(x)= \begin{cases}1, & |x| \leq x_{a}-1, \\ 0, & |x|>x_{a},\end{cases}
$$

$\left|g_{a}^{\prime}(x)\right| \leq 2$ and $\left|g_{a}^{\prime \prime}(x)\right| \leq 4$. Define

$$
u_{a}=u-g_{a} u_{x} /(4 a u) \text { and } v_{a}=u+g_{a} u_{x} /(4 a u) \text {. }
$$

Then

$$
P\left\{\left(u_{a}, v_{\alpha}\right)\right\}=u
$$

and

$$
P\left\{\left(a A+a^{2} B\right)\left(u_{a}, v_{\alpha}\right)\right\}=C u \text {. }
$$

Proof. The functions $g_{a}$ were selected so that $u_{a}$ and $v_{a}$ are nonnegative, and so that the dominated convergence theorem would imply (5.4).

Consider

$$
\begin{aligned}
& \left(a A+\alpha^{2} B\right)\left(u_{a}, v_{\alpha}\right) \\
& =\left(\alpha\left(g_{a}-1\right) u_{x}+\frac{1}{4} g_{a}^{\prime} \frac{u_{x}}{u}+\frac{1}{4} g_{a} \frac{d}{d x}\left(\frac{u_{x}}{u}\right),-a\left(g_{a}-1\right) u_{x}+\frac{1}{4} g_{a}^{\prime} \frac{u_{x}}{u}+\frac{1}{4} g_{a} \frac{d}{d x}\left(\frac{u_{x}}{u}\right)\right) .
\end{aligned}
$$

Since $\left|u_{x}\right|$ is bounded by $\left|M / x^{3}\right|$ for some $M$, we can bound the $L_{1}$ norm of the first term of each component by

$$
2 a \int_{x_{a}-1}^{\infty} \frac{M}{x^{3}} d x=\frac{a M}{\left(x_{a}-1\right)^{2}} .
$$

The conditions on $u$ imply $x_{a} \geq c a$ for some $c>0$ and $a$ sufficiently large, and hence this goes to zero as a goes to infinity. The limits of the other terms are straightforward and we have (5.5).

We must now check that Lemma (5.3) gives convergence for "sufficiently many" $u$. That is we must show that

$$
\left\{u-\beta 1 / 4\left(d^{2} / d x^{2}\right) \log u: u\right. \text { satisfies the conditions of Lemma (5.3)\} }
$$

is dense in $\left\{u \in L_{1}(\mathbf{R}): u \geq 0\right\}$ for all sufficiently small $\beta$. If $u-\left(d^{2} / d x^{2}\right) \log u=f$, 
$v(x)=u(a x)$ and $g(x)=f(a x)$ then $v-\left(1 / a^{2}\right)\left(d^{2} / d x^{2}\right) \log v=g$. Consequently it is sufficient to consider the case $\beta=4$.

Lemma (5.6). Let $f$ be nonnegative and continuous with compact support on $\mathbf{R}$, and not identically zero. Then there exists a solution of

$$
u-\left(d^{2} / d x^{2}\right) \log u=f
$$

satisfying the conditions of Lemma (5.3).

Proof. Since the form of the equation is translation invariant we may assume without loss of generality that $f(0)>0$. Let

$$
d=\int_{0}^{\infty} f(y) d y, \quad c=\int_{-\infty}^{0} f(y) d y .
$$

Select $a<-2 / c$ and $b>2 / d$ so that the support of $f$ is contained in $[a, b]$. Let $\boldsymbol{g}$ be a positive, continuous function satisfying

$$
\int_{0}^{x} g(y)=d-\frac{2}{x} \text { for } x>b
$$

and

$$
\int_{x}^{0} g(y)=c+\frac{2}{x} \text { for } x<a
$$

Define

For $x>b$

$$
\phi(x)=\exp \left\{-\int_{0}^{x} \int_{0}^{y}(f(z)-g(z)) d z d y\right\}
$$

$$
\phi(x)=\exp \left\{-\int_{0}^{b} \int_{0}^{y}(f(z)-g(z)) d z d y\right\} \exp \left\{-\int_{b}^{x} \frac{2}{y} d y\right\}=\frac{\text { const }}{x^{2}} .
$$

Similarly $\phi(x)=$ const $/ x^{2}$ for $x<a$. We are interested in finding a solution of (5.7) of the form $u(x)=k e^{w(x)} \phi(x)$ where $k$ is a constant which will be determined later. Substituting this form into the equation we obtain

$$
k e^{w} \phi-\left(d^{2} / d x^{2}\right) w-\left(d^{2} / d x^{2}\right) \log \phi=f .
$$

Considering the definition of $\phi$, this becomes

$$
k e^{w} \phi-\left(d^{2} / d x^{2}\right) w=g \text {. }
$$

Since $g>0$ and $g(x)=2 / x^{2}$ for $x>b$ and $x<a$ we note that $g / \phi$ is bounded above and bounded away from zero.

Let $M_{1}=\inf _{x} g(x) / \phi(x)$ and $M_{2}=\sup _{x} g(x) / \phi(x)$.

Select $0<\epsilon<1$ and $k>0$ such that

$$
M_{2} / k e>1, \quad \epsilon e^{-\epsilon}=\left(M_{2} / k e\right) \exp \left\{-M_{2} / k e\right\}, \quad \text { and } M_{1} / k \geq e^{\epsilon} .
$$

This is possible since making $k$ small forces $\epsilon$ to be small and $M_{1} / k$ large. 
Suppose $w$ is continuous and satisfies $\epsilon \leq w \leq M_{2} / k e$. The equation

$$
\left(k e^{w} \phi / w\right) v-\left(d^{2} / d x^{2}\right) v=g,
$$

has a unique bounded solution $v$ (see Appendix, Lemma (6.4)). Define $F(w)$ by $F(w)=v$. The solution $v$ is twice continuously differentiable and satisfies

$$
v \leq \sup _{x} w(x) e^{-w(x)} \frac{g(x)}{k \phi(x)} \leq \frac{M_{2}}{k} \sup _{z} z e^{-z}=\frac{M_{2}}{k e}
$$

and

$$
v \geq \inf _{x} w(x) e^{-w(x)} \frac{g(x)}{k \phi(x)} \geq \epsilon e^{-\epsilon} \frac{M_{1}}{k} \geq \epsilon .
$$

Let $\Gamma=\left\{w: w\right.$ is continuous and $\left.\epsilon \leq w \leq M_{2} / k e\right\}$. Then $F: \Gamma \rightarrow \Gamma$ and is continuous in the topology of uniform convergence on compact sets. Furthermore

$$
\left|v_{x}(z)-v_{x}(y)\right|=\left|\int_{y}^{z} \frac{d^{2}}{d x^{2}} v d x\right| \leq k \frac{e^{\epsilon}}{\epsilon} \frac{M_{2}}{k e} \int_{y}^{z} \phi d x+\int_{y}^{z} g d x .
$$

Since $\phi$ and $g$ are in $L_{1}(R)$ it follows that $F$ maps $\Gamma$ into a compact subset of $\Gamma$ and hence has a fixed point $w$. But a fixed point of the mapping $F$ is a solution of (5.9).

We now must verify that $u=k e^{w} \phi$ satisfies the conditions of Lemma (5.3). Since $\epsilon \leq w \leq M_{2} / k e$ the fact that $c_{1} / x^{2} \leq u \leq c_{2} / x^{2}$ for some $c_{1}$ and $c_{2}$ and $|x|$ sufficiently large follows from the definition of $\phi$.

Consider $u_{x}=k e^{w} \phi_{x}+k e^{w} \phi w_{x}$. Again, from the definition of $\phi$, in order to verify $\left|u_{x}\right| \leq\left|M / x^{3}\right|$ for some $M$ it is sufficient to show that $x w w_{x}$ is bounded.

From $(5.11)$ it is clear that $\lim _{x \rightarrow \infty} w_{x}(x)$ exists and the boundedness of $w$ implies the limit is zero. Therefore

$$
w_{x}(x)=\int_{x}^{\infty}\left(g-k e^{w} \phi\right) d x .
$$

But $g-k e^{w} \phi=O\left(1 / x^{2}\right)$, and hence $w_{x}(x)=O(1 / x)$ for $x \rightarrow \infty$. Similarly $w_{x}(x)=$ $O(1 / x)$ for $x \rightarrow-\infty$.

Remark. General results of this type are considered in the linear case in [6]. General material on discrete velocity models for the Boltzmann equation as well as some results related to the above for a different model can be found in [13].

The author would like to thank Professor Howard Conner and Professor Michael Crandall for a number of helpful conversations.

6. Appendix. We verify that the operators considered in $\$ 5$ are dissipative.

Lemma (6.1). Let $L=L_{1}(\mathbf{R}) \times L_{1}(\mathbf{R})$ with $\|(u, v)\|=\|u\|_{L_{1}}+\|v\|_{L_{1}}$, and let $L^{+}=\{(u, v) \in L: u \geq 0, v \geq 0\}$. Let $B(u, v)=\left(v^{2}-u^{2}, u^{2}-v^{2}\right)$ with $\mathscr{D}(B)=$ $\left\{(u, v) \in L^{+}: u^{2}, v^{2} \in L_{1}(\mathrm{R})\right\}$. Then $B$ is dissipative. 
Proof.

$$
\begin{aligned}
\|\left(u_{1}, v_{1}\right)-\left(u_{2},\right. & \left.v_{2}\right)-a\left(B\left(u_{1}, v_{1}\right)-B\left(u_{2}, v_{2}\right)\right) \| \\
= & \int\left|u_{1}-u_{2}-a\left(v_{1}^{2}-v_{2}^{2}-\left(u_{1}^{2}-u_{2}^{2}\right)\right)\right| d x \\
& +\int\left|v_{1}-v_{2}-a\left(u_{1}^{2}-u_{2}^{2}-\left(v_{1}^{2}-v_{2}^{2}\right)\right)\right| d x \\
\geq & \int\left[\left(1+a\left(u_{1}+u_{2}\right)\right)\left|u_{1}-u_{2}\right|-\alpha\left(v_{1}+v_{2}\right)\left|v_{1}-v_{2}\right|\right] d x \\
& +\int\left[\left(1+\alpha\left(v_{1}+v_{2}\right)\right)\left|v_{1}-v_{2}\right|-a\left(u_{1}+u_{2}\right)\left|u_{1}-u_{2}\right|\right] d x \\
= & \int\left|u_{1}-u_{2}\right| d x+\int\left|v_{1}-v_{2}\right| d x=\left\|\left(u_{1}, v_{1}\right)-\left(u_{2}, v_{2}\right)\right\| .
\end{aligned}
$$

Since $A$ is dissipative and generates a linear semigroup, it follows that $A+$ $a B$ is dissipative.

The fact that $C$ is dissipative for functions satisfying the conditions of Lemma (5.3) follows from the fact that $A+a B$ is dissipative.

However, we will verify that $C$ is dissipative on all of $\mathscr{D}(C)$ as defined in Theorem (5.1).

Lemma (6.2). Let $C$ be as defined in Theorem (5.1). Then $C$ is dissipative.

Proof. Let $u_{0} v \in \mathscr{D}(C)$ and let $S_{1}$ be the collection of intervals $I=(a, b)$ such that $u-v>0$ on $I$ and either $a=-\infty$ or $u(a)=v(a)$ and either $b=\infty$ or $u(b)=v(b)$. Let $\delta_{2}$ be the similar collection of intervals on which $u-v<0$. For $I \in S_{1}, u(a)=v(a)$ implies $u_{x}(a)-v_{x}(a) \geq 0$ and $u(b)=v(b)$ implies $u_{x}(b)-$ $v_{x}(b) \leq 0$.

We then have

$$
\begin{aligned}
& \int\left|u-v-a\left(\frac{d^{2}}{d x^{2}} \log u-\frac{d^{2}}{d x^{2}} \log v\right)\right| d x \\
& \quad \geq \int|u-v| d x-a \int \operatorname{sign}(u-v)\left(\frac{d^{2}}{d x^{2}} \log u-\frac{d^{2}}{d x^{2}} \log v\right) d x \\
& =\int|u-v| d x+a \sum_{I \in \delta_{1}}-\int_{I}\left(\frac{d^{2}}{d x^{2}} \log u-\frac{d^{2}}{d x^{2}} \log v\right) d x \\
& +a \sum_{I \in \delta_{2}} \int_{I}\left(\frac{d^{2}}{d x^{2}} \log u-\frac{d^{2}}{d x^{2}} \log v\right) d x .
\end{aligned}
$$

Suppose, for example, $l=(a, b) \in \mathcal{S}_{1}, a \neq-\infty$ and $b=\infty$. The fact that $\lim _{x \rightarrow \infty} u_{x} / u$ $=\lim _{x \rightarrow \infty} v_{x} / v=0$ implies 


$$
\begin{aligned}
-\int_{a}^{b}\left(\frac{d^{2}}{d x^{2}} \log u-\frac{d^{2}}{d x^{2}} \log v\right) d x & =\left(\frac{u_{x}(a)}{u(a)}-\frac{v_{x}(a)}{v(a)}\right) \\
& =\frac{u_{x}(a)-v_{x}(a)}{u(a)} \geq 0 .
\end{aligned}
$$

It follows in a similar manner that all terms in the summations on the righthand side of (6.3) are nonnegative, and hence it is bounded below by $\int|u-v| d x$.

Lemma (6.4). Suppose $\gamma(x)$ and $g(x)$ are nonnegative, bounded and continuous and that there exists $\epsilon>0$ such that $\gamma(x) \geq \epsilon$ for all $x$. Then

$$
\gamma v-1 / 2 v^{\prime \prime}=g
$$

bas a unique, bounded solution and

$$
\inf _{x} \frac{g(x)}{\gamma(x)} \leq v \leq \sup _{x} \frac{g(x)}{\gamma(x)}
$$

Proof. The uniqueness follows from the fact that the only bounded solution of $v^{\prime \prime}=\gamma v$ is $v \equiv 0$.

Since the author is a probabilist, to obtain existence we appeal to results conceming Brownian motion. (See Dynkin [15, p. 46].) In particular if $X(t)$ is standard Brownian motion, $(X(0)=0, \operatorname{Var}(X(t))=t)$ then the solution of $(6.5)$ is

$$
v(x)=E\left(\int_{0}^{\infty} \exp \left\{-\int_{0}^{t} \gamma(x+X(s))\right\}_{g}(x+X(t)) d t\right) .
$$

The inequalities in (6.6) follow immediately by making the change of variable $\tau=\int_{0}^{t} \gamma(x+X(s))$ in (6.7).

\section{REFERENCES}

1. H. Brezis and A. Pazy, Convergence and approximation of semigroups of nonlinear operators in Banach spaces, J. Functional Analysis 9 (1972), 63-74. MR 45 \#2529.

2. Howard E. Conner, Some general properties of a class of semilinear hyperbolic systems analogous to the differential-integral equations of gas dynamics, J. Differential Equations 10 (1971), 188-203. MR 44 \#7130.

3. M. G. Crandall and T. M. Liggett, Generation of semigroups of nonlinear transformations on general Banach spaces, Amer. J. Math. 93 (1971), 265-298. MR 44 \#4563.

4. Thomas G. Kurtz, Extensions of Trotter's operator semigraup approximation theorems, J. Functional Analysis 3 (1969), 354-375. MR 39 \#3351.

5. - A general theorem on the convergence of operator semigroups, Trans. Amer. Math. Soc. 148 (1970), 23-32. MR 41 \#867.

6. —_, A limit theorem for perturbed operator semigroups with applications to random evolutions, J. Functional Analysis 12 (1973), 55-67. 
7. Joel L. Mermin, An exponential limit formula for nonlinear semigroups, Trans. Amer. Math. Soc. 150 (1970), 469-476. MR 41 \#7478.

8. Isao Miyadera, $O_{n}$ the convergence of nonlinear semigroups, Tohoku Math. J. (2) 21 (1969), 221-236. MR 40 \#790.

9. _-, On the convergence of nonlinear semigroups. II, J. Math. Soc. Japan 21 (1969), 403-412. MR 40 \# 791.

10. Isao Miyadera and Ôharu Shinnosuke, Approximation of semigroups of nonlinear operators, Tohoku Math. J. (2) 22 (1970), 24-47. MR 41 \#749.

11. Mark A. Pinsky, Differential equations with a small parameter and the central limit theorem for functions defined on a finite Markov chain, $\mathrm{Z}$. Wahrscheinlichkeitstheorie und Verw. Gebiete 9 (1968), 101-111. MR 37 \#3651.

12. H. F. Trotter, Approximation of semigroups of operators, Pacific J. Math. 8 (1958), 887-919. MR 21 \#2190.

13. S. K. Godunov and U. M. Sultangazin, Discrete models of the kinetic Boltzman equation, Uspehi Mat. Nauk 26 (1971), no. 3 (159), 3-51 = Russian Math. Surveys 26 (1971), no. 3, 1-56. MR 45 \#3027.

14. Jerome A. Goldstein, Approximation of nonlinear semigroups and evolution equations, J. Math. Soc. Japan 24 (1972), 558-573.

15. E. B. Dynkin, Markov processes. II, Fizmatgiz, Moscow, 1963; English transl., Die Grundlehren der math. Wissenschaften, Band 122, Academic Press, New York; SpringerVerlag, Berlin, 1965. MR 33 \# 1887.

DEPARTMENT OF MATHEMATICS, UNIVERSITY OF WISCONSIN, MADISON, WISCONSIN 53706 